\title{
AT Humboldt 2000 (Team Description)
}

\author{
Hans-Dieter Burkhard, Joscha Bach, Kay Schröter, Jan Wendler*, \\ Michael Gollin, Thomas Meinert, and Gerd Sander \\ Humboldt University Berlin, Department of Computer Science, \\ Artificial Intelligence Laboratory, D-10099 Berlin, Germany \\ $\{\mathrm{hdb}$, bach, kschroet, wendler, gollin, meinert, sander\}@informatik.hu-berlin.de \\ http://www.ki.informatik.hu-berlin.de
}

\section{Introduction}

Our agent team AT Humboldt 2000 is partly an extension of our former team AT Humboldt 99[2,3]. Again we used a BDI architecture. Especially the world model and some skills where revised. A new timing concept and a completely different architecture for the deliberation component were developed. The actual development was subject of an undergraduate course. Because of problems with the integration of components developed by different work groups, we were forced to start in RoboCup 2000 with a mixed team, consisting mainly of an extended version of the players used in EuRoboCup 2000. Only the goalie used all our new concepts.

\section{Special Team Features}

We are interested in virtual soccer for the development and the evaluation of our research topics in artificial intelligence, which concern the fields of Agent oriented techniques, Multi-Agent Systems and Case Based Reasoning. Thus many aspects of our soccer program are heavily influenced by these fields. Key feature of our actual agent concept is a deliberation structure that allows for reasoning and evaluations persisting over several simulation steps, while low-level behaviours (skills) are continuously executed (in the form of plans), as long as neither the deliberator nor conditions in the environment of the agent call for a change of action. The deliberator is going to be extended to allow for high-level descriptions of situations and orchestrated action.

\section{World Model}

AT Humboldt 2000 uses the same world model concept as in our previous teams[1-4] (plus several serious bugs that impair the performance of the basic

\footnotetext{
* This work has been partially supported by the German Research Society, BerlinBrandenburg Graduate School in Distributed Information Systems (DFG grant no. GRK 316).
} 
skills). For any given time a situation object will be generated which consists of object representions for teammates, opponent players, the ball and the agent itself. Flags are only used to determine the own absolute object representation which consists of the absolute player position, speed, body direction and of the relative face direction. With this data the absolute objects, representations of the other players and the ball are calculated. The agent can get a new situationobject from sensory information. Another way to get a new situation-object is the simulation of the actions of the agent, like it is done in the SoccerServer. So after every sensor information we have two concurrent Situation-objects which are merged together by finding corresponding players and using the best information of both situations for the new one. Reliability of information is currently only assessed by time-stamps.

\section{Communication}

Though rarely used, the agents are able to communicate by means of compressed strings of data, consisting of world-model and status/intention information. To ensure the integrity of the data, the string is time-stamped and check-summed.

\section{$5 \quad$ Skills}

AT Humboldt 2000 makes use of a new kick skill with adjustable start and target speeds and avoidance of nearby enemy players. Kick actions consist of one to four kick commands and are calculated, not learned. Tests using a correct world model have shown that accurate kicks of $2.4 \mathrm{~m} / \mathrm{s}$ were consistently achieved. Because of our malfunctioning world model, the new kicking skill turned out to be of very little use and did not show any practical improvement over the old one during RoboCup2000. Consequently, dribbling and passing that should capitalize on better kicks, did not improve, while goal kicking got more powerful.

\section{Strategy}

Our agent architecture uses a mental deliberation structure which is best described by a belief-desire-intention architecture (BDI) [5]. Distinct from other (e.g. logically motivated) approaches our approach is closely related to procedural thinking, and we use object oriented programming for the implementation.

To cope with the increasing number of different intentions, a concept of nested intentions was choosen[6]. The design allows for high-level descriptions of plans and strategies (i.e. double passes, clique playing etc.) to combine preadjusted with emerging behaviour, a process we consider as necessary to adapt to strategic features and weaknesses of opponent teams. AT Humboldt 2000, however, failed to pose any real threat for advanced opponents, mainly because of its poor basic skills. 


\section{Team Development}

The virtual soccer team AT Humboldt is developed by our AI group. Most of the work is done by students. As in the previous years some tasks are practical excercises for an AI course during summer semester. Other aspects are examined in diploma theses. A small core development group maintains the code and coordinates the work. Besides the experiments with AI methods, the project is also a challenge for software development: The sources of AT Humboldt contain over 40.000 lines of code. It is a non trivial task to integrate new ideas during extremly short intervals.

To support the concurrent development we use the freely available source code management system CVS[7] and the documentation system doc $++[8]$. We have developed an extensive testing and debugging tool - the logbrowser. It controls the log player and synchronously displays information on the agents. Information from different components of our agents can be selected or deselected for displaying, as desired. More efficient logging mechanisms and a compressed log format make it possible to write detailed logs of all agents at real time conditions, which turned out to be very helpful in the cases where it was consequently put to use.

Team Leader: Prof. Hans-Dieter Burkhard

Team Members:

Prof. Hans-Dieter Burkhard (professor, leader of the AI group)

Joscha Bach, Kay Schröter (research assistants)

Jan Wendler (PhD student)

Michael Gollin (undergraduate student)

- Humboldt-University Berlin, Germany

- did attend the competition

Thomas Meinert, Gerd Sander (undergraduate students)

- Humboldt-University Berlin, Germany

- did not attend the competition

Web page http://www.ki.informatik.hu-berlin.de/

\section{Conclusion}

The main goals of our further work are the extension and the refinement of the new deliberation concept, the development of a new world model, the usage of learning and CBR techniques to evaluate situations and preselect useful intentions, and the modelling of opponents in the frame of the BDI architecture to anticipate their behaviour.

Furthermore we want to support our sony legged robot team, the Humboldt Hereos, with techniques already used by our simulation team. 


\section{References}

1. H.-D. Burkhard, M. Hannebauer, and J. Wendler. AT Humboldt - Development, Practice and Theory. In H. Kitano, editor, RoboCup-97: Robot Soccer World Cup $I$, volume 1395 of $L N A I$, pages 357-372. Springer Verlag, 1998.

2. H.-D. Burkhard, M. Hannebauer, J. Wendler, H. Myritz, G. Sander, and T. Meinert. BDI Design Principles and Cooperative Implementation - A Report on RoboCup Agents. In Proceedings of the IJCAI-99 Third International Workshop on RoboCup, Stockholm, Schweden, Aug. 1999.

3. H.-D. Burkhard, J. Wendler, T. Meinert, H. Myritz, and G. Sander. AT Humboldt in RoboCup-99. In M. Veloso, E. Pagello, and H. Kitano, editors, RoboCup-99: Robot Soccer World Cup III, LNAI. Springer Verlag, 2000.

4. P. Gugenberger, J. Wendler, K. Schröter, and H.-D. Burkhard. AT Humboldt in RoboCup-98. In M. Asada and H. Kitano, editors, RoboCup-98: Robot Soccer World Cup II, volume 1604 of LNAI, pages 358-363. Springer Verlag, 1999.

5. A. S. Rao and M. P. Georgeff. BDI agents: From theory to practice. In V. Lesser, editor, Proceedings of the ICMAS-95, pages 312-319. MIT Press, 1995.

6. G. Sander The Intention-Agent: a new approach for a solution of longterm cooperation in RoboCup. In H.-D. Burkhard, L. Czaja, A. Skowron, P. Starke, editors, Proceedings of the Workshop on Concurrency, Specification and Programming 2000, Informatik-Bericht 140(2), pages 235-239. Humboldt-University, 1995.

7. CVS: http://www.loria.fr/ molli/cvs-index.html

8. DOC++: http://www.zib.de/Visual/software/doc++/index.html 